\title{
Bloch gain in quantum cascade lasers
}

\author{
ROMAIN TERAZZ ${ }^{1 * \dagger}$, TOBIAS GRESCH ${ }^{1 *}$, MARCELLA GIOVANNINI ${ }^{1}$, NICOLAS HOYLER ${ }^{1}$, \\ NORIHIKO SEKINE² AND JÉRÔME FAIST ${ }^{1}$
}

\author{
${ }^{1}$ Institute of Physics, University of Neuchâtel, A.-L. Breguet 1, 2000 Neuchâtel, Switzerland \\ ${ }^{2}$ Institute of Industrial Science, University of Tokyo, 4-6-1 Meguro-ku, Komaba, Tokyo 153-8505, Japan \\ *These authors contributed equally to this work \\ †e-mail: romain.terazzi@unine.ch
}

Published online: 1 April 2007; doi:10.1038/nphys577

Esaki and Tsu's superlattice ${ }^{1}$, made by alternating two different semiconductor materials, was the first one-dimensional artificial crystal that demonstrated the ability to tailor semiconductor properties. One motivation of this work was the realization of the Bloch oscillator ${ }^{2,3}$ and the use of its particular dispersive optical gain $^{4,5}$ to achieve a tuneable source of electromagnetic radiation. However, these superlattices were electrically unstable in the steady state $^{6}$. Fortunately, because it is based on scatteringassisted transitions, this particular gain does not arise only in superlattices, but also more generally in semiconductor heterostructures ${ }^{7,8}$ such as quantum cascade lasers ${ }^{9}$ (QCLs), where the electrical stability can be controlled ${ }^{10}$. Here, we show the unambiguous spectral signature of Bloch gain in a special QCL designed to enhance the latter by exhibiting laser action in the condition of weak to vanishing population inversion.

In solids, electrons have a fixed relation between momentum and energy: they move along energy bands, as known from condensed-matter theory. When an electric field is applied they are accelerated but the lattice forces a periodic motion at a definite Bloch frequency. This phenomenon is known as Bloch oscillations, and the idea was successfully used by Zener to explain the dielectric breakdown ${ }^{3}$. However, in usual solids the strong scattering due to impurities and carrier-carrier interaction prevents the observation of such oscillations, as the lattice constant is too short to allow the electrons to complete even one oscillation cycle. In superlattices, the lattice constant can be chosen and a subtle engineering may allow electrons to achieve a few oscillations before scattering. As this phenomenon is fascinating from a condensed-matter point of view, it also opens new perspectives for optics because charge oscillations naturally couple to radiation and offer a way to emit coherent radiation.

Therefore, the important question is whether these oscillations can be self-sustained and provide optical gain. First, Ktitorov ${ }^{4}$ and then Ignatov and Romanov ${ }^{5}$ addressed the problem theoretically with Boltzmann equations and succeeded in providing a definitive signature for Bloch oscillations in superlattices in terms of a particular spectral response: the Bloch oscillations are found to amplify the electromagnetic field (optical gain) on the low-energy side of the oscillation frequency, whereas they absorb photons on the high-energy side. This particular shaped gain-Bloch gain-is the main feature of the Bloch oscillator. A series of experiments ${ }^{11-13}$ using pulsed ultrafast techniques have successfully shown the existence of Bloch oscillations as electrons are pumped in a higher energy band and collectively oscillate over their dephasing time. However, the Bloch gain extends to zero frequencies and the structure becomes unstable in the steady state, so far preventing the observation of net gain in superlattices, although some evidence in photocurrent ${ }^{14}$ and more recently in absorption ${ }^{15}$ were demonstrated by driving superlattices with a strong terahertz field.

Recently, Bloch gain was investigated from a pure quantummechanical perspective in a density matrix formalism, as reported by Willenberg et $a l^{7}$, and also in a Green function formalism, as reported by Wacker ${ }^{8}$. These theoretical studies demonstrated that Bloch gain can arise between any pair of states, called subbands, in a semiconductor heterostructure and that the latter does not need to be a superlattice. As shown in Fig. 1a, the origin of this gain is second-order, scattering-assisted, optical transitions occurring between pairs of subbands. In an emission process, electrons at wavevector $\mathbf{k}$ in the upper subband undergo a vertical optical transition to a virtual lower subband, and then scatter elastically to a real state at wavevector $\mathbf{k}^{\prime}$. The absorption process proceeds in a symmetric manner, exchanging the upper and lower subbands Fig. 1b. The essential feature of these second-order processes is that they connect states with different wavevectors. As a consequence, as compared with direct transitions, the populationinversion requirement for these second-order processes is relaxed, as population inversion is only necessary between states with different wavevectors and not globally between subbands. For example, in the particular case where both subbands have the same populations, gain is achieved for photon energies smaller than the transition energy as the occupation at the final state $\mathbf{k}^{\prime}$ is automatically smaller than the one of the initial state $\mathbf{k}$. Note that because of the short upper-state lifetime in quantum cascade lasers (QCLs), populations and occupations at typical electron temperatures are small, and therefore Pauli blocking is negligible although it is implicitly contained in the theoretical models. As these theories do not require any specific shape for the heterostructure potential, Bloch gain may also be observed in a sample where an injection region has been inserted between active wells to stabilize the structure electrically, as is routinely done in QCLs ${ }^{9}$. Moreover, the model could treat subbands with arbitrary populations, from perfect inversion (no lower-state population) and equal population (in which case it was shown numerically to yield the same results as Boltzmann models), to absorption (no upper-state population) and therefore this second-order picture generalizes the concept of gain in semiconductor heterostructures.

To demonstrate experimentally the existence of Bloch gain in QCL structures while excluding other phenomena that were predicted to also yield gain without global population inversion between subbands, such as non-reciprocity ${ }^{16}$ and local $k$-space 

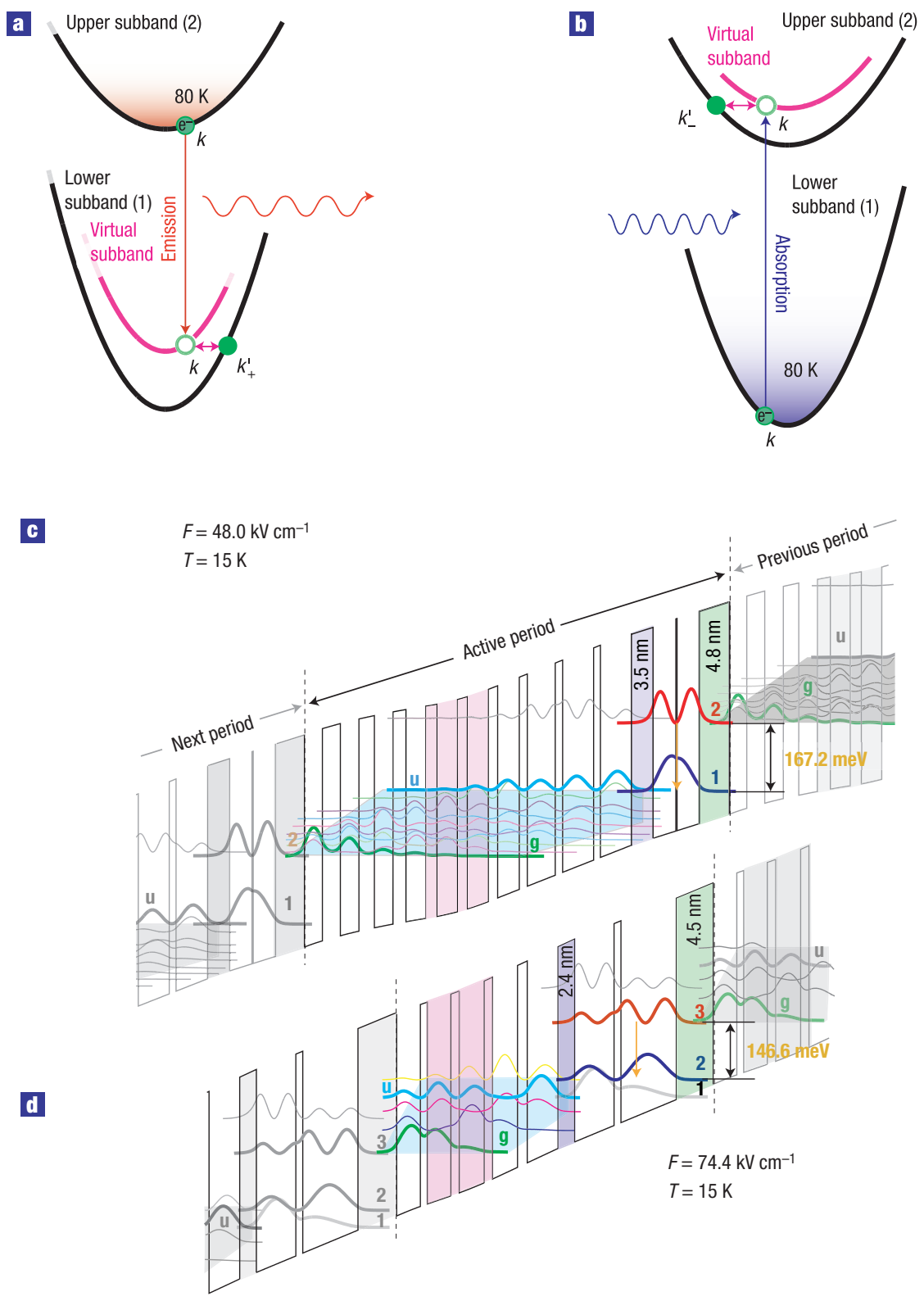

Figure 1 Bloch gain mechanism and samples details. a,b, Scattering-assisted optical transitions between a pair of subbands with conduction band non-parabolicity. The black parabola represent real-energy states and the pink parabola are virtual states dressed by scattering. c,d, Layer sequences and band structure of the investigated samples. Each structure is shown at the injection resonance field. The layer sequence starts from the injection barrier and the thicknesses are in nanometres; roman, respectively bold, numbers indicate $\mathrm{In}_{0.53} \mathrm{Ga}_{0.47} \mathrm{As}$, respectively $\mathrm{Al}_{0.48} \mathrm{In}_{0.52} \mathrm{As}$ alloy, acting as well, respectively barrier material. Underlined layers are $3 \times 10^{17} \mathrm{~cm}^{-3} \mathrm{Si}$ doped. c, N258 single-quantum-well structure with layers, from right to left: 4.8/3.6/0.2/3.6/3.5/5.1/1.1/5.0/1.2/4.5/1.3/3.5/1.5/ 3.4/ 1.6/ 3.3/ 1.8/3.2/2.1/3.0/2.5/3.0/2.9/2.9. Nominal sheet carrier density is $3.03 \times 10^{11} \mathrm{~cm}^{-2}$. Period length is $68.6 \mathrm{~nm}$, repeated 35 times. d, N123 two-well structure with layers, from right to left: 4.5/8.0/1.0/5.7/2.4/4.4/1.4/3.6/ 1.2/ 3.6/ 1.2/ 3.4/1.0/3.4. Nominal sheet carrier density is $2.82 \times 10^{11} \mathrm{~cm}^{-2}$. Period length is $48.4 \mathrm{~nm}$, repeated 35 times.

inversion due to non-parabolicity ${ }^{17,18}$, a spectrally resolved gain measurement is carried out in a specially designed QCL. On the basis of a comparison of laser characteristics, laser operation without global population inversion between subbands was already reported $^{17}$ and was attributed to the combined effect of nonparabolicity and a hot-electron distribution in the lower state ${ }^{18}$. However, no measurement of the gain curve was carried out in this experiment, preventing a definitive interpretation of these results.

Here, in contrast to QCLs that are usually optimized to ensure a strong population inversion, our structure was designed to enable the population inversion to be varied over a large range. To this end, as shown in Fig. 1b,c, the active period consists of an injection region (shaded area) coupled to an active quantum well by an injection and extraction tunnel barrier. Under the designed bias of $48 \mathrm{kV} \mathrm{cm}^{-1}$, the band structure is aligned so that electrons tunnel resonantly from the injector ground state $|g\rangle$ of the previous period to the upper lasing state $|2\rangle$. As shown in ref. 10 , the thickness of the injection barrier must be optimized: in the low-coupling regime (thick injection barrier), the maximum current that can be injected in the upper state is proportional to the coupling strength; 

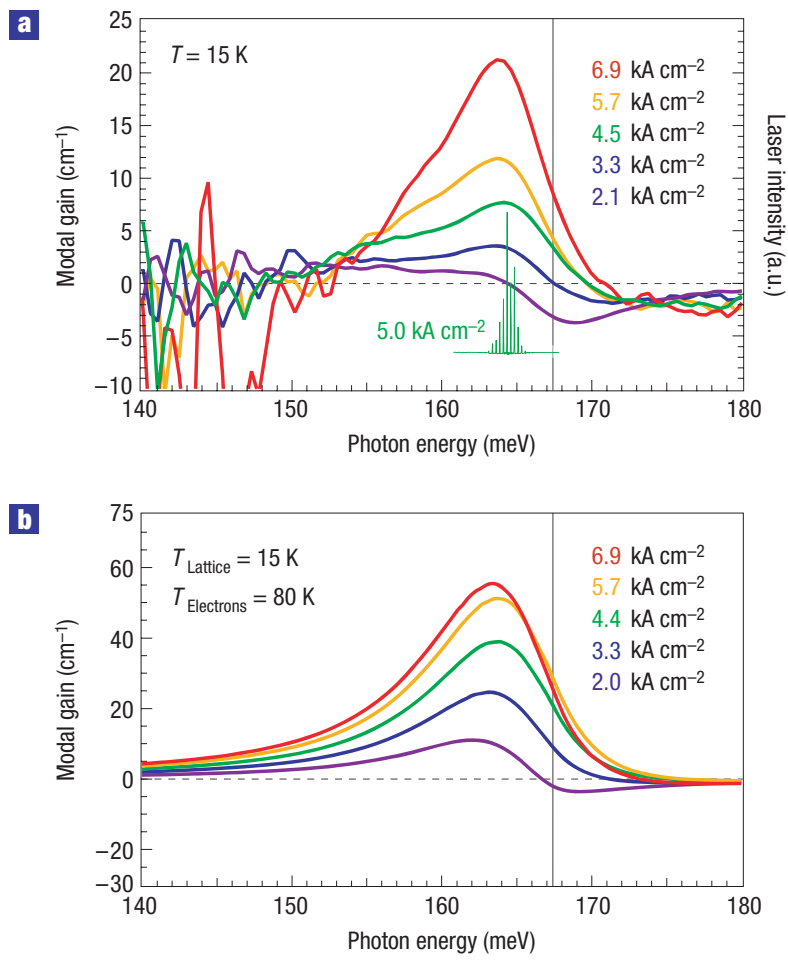

Figure 2 Results for Bloch gain sample (N258). a, Net modal gain measurements achieved for increasing current densities in the amplifier section. $\mathbf{b}$, Simulations. The vertical line connecting the top and bottom axes represents the transition energy computed from the band structure between the subband edges ( $k=0$ states) in the active well. The spectrum of device N258 operated as a laser is also shown in a.

in the opposite (thin injection barrier), the maximum current is only limited by the upper-state lifetime but leakage currents are then proportional to the coupling. Downfield of the active well, an extraction barrier allows us to control the population of the lower lasing state $|1\rangle$ : because $|1\rangle$ is the ground state of the active well, coupling to the upper state $|u\rangle$ of the injector region is achieved by tunnelling of electrons through the barrier; by varying the width thereof, the extraction time and therefore the population of the lower state can be tuned. A submonolayer, 0.2-nm-thick AlInAs barrier, inserted in the active quantum well, raises the energy of the lower state by about $18 \mathrm{meV}$, so that it faces the extraction miniband. The presence of this barrier also enhances the elastic broadening of the lower state.

A systematic study of injection and extraction barrier widths has been carried out, allowing us to correlate the characteristics of the device, as well as the shape of the gain curve, with the lowerstate lifetime. As expected, a reduction of the population inversion is observed when the extraction barrier width is increased, translating into an increase of the laser threshold. Here, we choose to focus our study on sample N258, which achieved the lowest population inversion while still showing laser action. As a reference, we also studied a sample based on a coupled well where a strong population inversion is expected ${ }^{19}$. As shown in Fig. 1d, the design of sample N123 relies on a strong depopulation of the lower lasing state $|2\rangle$ through an intersubband longitudinal optical phonon resonance $(32 \mathrm{meV})$ between $|2\rangle$ and $|1\rangle$, which keeps the lasing doublet $|3\rangle,|2\rangle$ in a strong population-inversion regime.

The measurements, carried out at liquid helium temperature, are shown for the single-quantum-well sample (N258) as a function
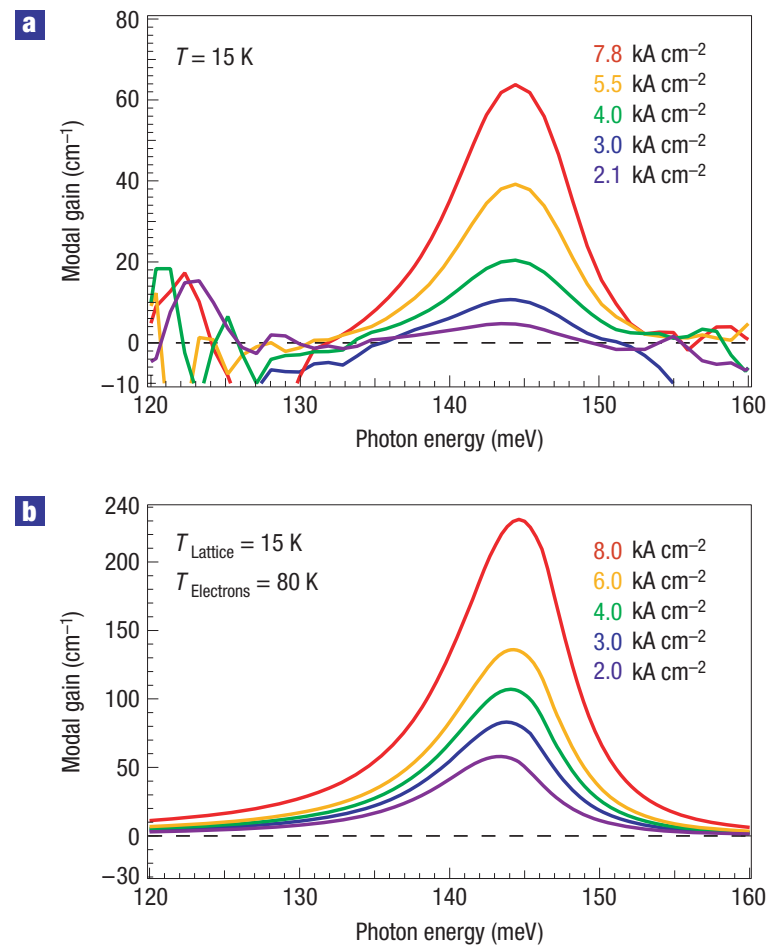

Figure 3 Results for the reference sample (N123). a, Net modal gain measurements achieved for increasing current densities in the amplifier section. b, Simulations.

of injected current in Fig. 2a. At the lower current densities (2.1 and $3.3 \mathrm{kA} \mathrm{cm}^{-2}$ ), the gain shows a clear dispersive shape, with gain for photon energies below the expected transition energy and loss at higher energies. As the current is increased, a more symmetric shape is recovered, indicating an improvement in the population inversion as the injection is made more resonant.

Gain measurements of the control sample N123 are shown in Fig. 3a. As expected, the shape of the gain curve is almost symmetric for the whole range of injected current. For a given injection current density, the maximum gain is also larger by a factor of four. Such a behaviour is also predicted by the theoretical model.

The dispersive shape of the gain spectrum, and its dependence on the active-region design, are a clear demonstration of the presence of Bloch gain in the low-inversion sample. An absorption artefact that could simulate the dispersive shape shown by sample N258 would be measured equally in both samples if it was process or impurity related. In addition, careful computation of the absorption in the injector region for varying temperature and biases (see the Supplementary Information) may safely exclude the presence of a parasitic absorption line at such photon energy. As an additional check, the device N258 was then cleaved and operated as a laser; as expected its spectrum is centred at the photon energy at which the maximum of the gain was recorded, as shown in Fig. 2a.

The validity of our interpretation is further verified by computing the gain theoretically. The result of this computation is shown in Fig. 2b for sample N258 and in Fig. 3b for the reference sample N123. For the comparison to be meaningful, a procedure that used essentially no fitting parameters was implemented. The shape of the experimental and theoretical curves in Fig. 2 are found to be in good agreement. In comparison, the prediction for sample N123 Fig. $3 \mathrm{~b}$ shows a line that is much more symmetrical and closer to a lorenzian shape, again mirroring the experimental 
a
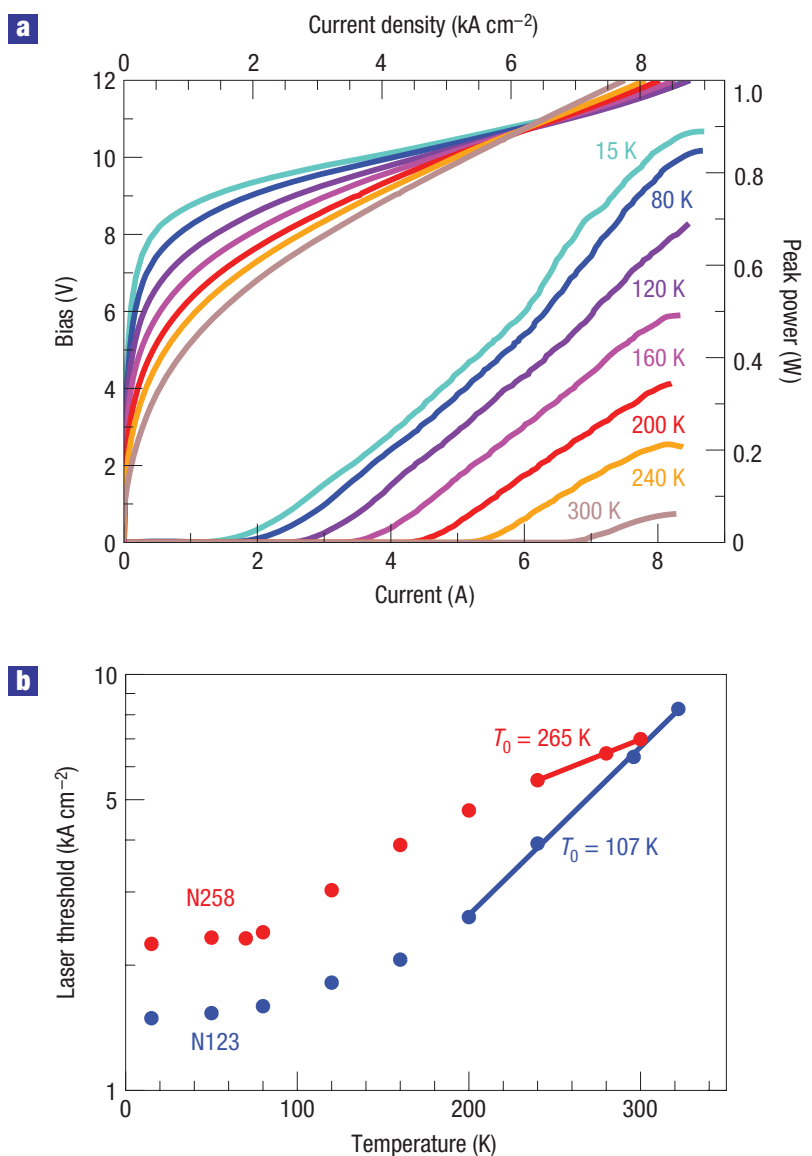

Figure 4 Temperature dependence of laser characteristics (N258) and laser threshold (N258, N123). a, Bias (left axis) and peak optical power (right axis) as a function of temperature for the single-quantum-well sample N258. $\mathbf{b}$, Threshold current density as a function of temperature for sample N258 $(3.8 \mathrm{~mm}$ long) and the reference sample $\mathrm{N} 123$ (2.6 $\mathrm{mm}$ long). The segments show the temperature range over which the $T_{0}$ parameter was fitted.

results. Although, for both samples, the gain shapes are in a good agreement with the experimental data, the absolute value of the gain is overestimated by a factor of three in each case. We attribute a part of this discrepancy to the experimental technique used to measure the gain spectra: parasitic light, emitted by the lightbulb section A, travelling through the substrate and collected by the lens will increase the intensity of the measured light for the normalization, in effect leading to an underestimation of the gain. Experimental evidence for this effect is obtained by comparing the waveguide losses measured with the multisection technique $\left(5 \mathrm{~cm}^{-1}\right)$ with a systematic measurement of the threshold current as a function of cavity length $\left(11 \mathrm{~cm}^{-1}\right)$. The latter value is also in good agreement with the computed one using a Drude model for free-carrier absorption. On the theoretical model side, neglecting all other scattering processes except for optical phonon scattering leads to an overestimate of the gain.

The magnitude of the gain measured in this work is much larger than the one predicted only for non-parabolicity ${ }^{18}$ and the shape of the gain curve is clearly different. In our model, although inclusion of the non-parabolicity was important to achieve a good correspondence between the shape of the experimental data and the theory, the key ingredient needed to predict both the dispersive shape and the right magnitude of the gain curve is the Bloch component of the second-order gain and not the non-parabolicity.

Lasers were also fabricated from sample N258 and N123 and their optical and electrical characteristics were measured as a function of temperature, as shown in Fig. 4. The threshold current density of sample $\mathrm{N} 258$, at low temperature, is $2.2 \mathrm{kA} \mathrm{cm}^{-2}$ for a $3.8-\mathrm{mm}$-long and $26-\mu \mathrm{m}$-wide device. Even allowing some uncertainty on the current values shown in Fig. 2 because of current spreading, the device clearly operates in the regime of Bloch gain, as inspection of the gain curve measured at $J=3.3 \mathrm{kA} \mathrm{cm}^{-2}$ shows a strong dispersive component. The device operates up to a maximum temperature larger than $300 \mathrm{~K}$, with a weak temperature dependence of the threshold current density that better fits a linear rather than the usual exponential dependence $J=J_{0} \exp \left(T / T_{0}\right)$. Nevertheless, fitting the data for $T>240 \mathrm{~K}$ with this expression yields $T_{0}=265 \mathrm{~K}$. In contrast, sample N123 exhibits the usual exponential dependence of the threshold current density with a value of $T_{0}=107 \mathrm{~K}$, as also reported in ref. 19 . Although more detailed temperature-dependent measurements and modelling would be required to rigorously prove that the temperature behaviour is indeed related to the nature of the gain, a weaker temperature dependence of the threshold current density is qualitatively expected from a device based on a Bloch gain mechanism. The threshold current density at $300 \mathrm{~K}$ of N258 is a factor of about five larger than recent results achieved in optimized structures, yet the level of performance of sample N258 is still surprising as its doping level and injection barrier thickness were optimized for the visualization of Bloch gain and not for sheer performance.

\section{METHODS}

The samples were grown using the InGaAs/AlInAs material system lattice matched on InP substrate and consisted of 35 periods of the injector/active region pair placed in the centre of an optical waveguide. As indicated in the caption of Fig. 1, a few layers in the centre of the injectors were Si doped to yield a sheet density of $n=3 \times 10^{11} \mathrm{~cm}^{-2}$ per period. Samples were then processed into laser ridges using wet etching, $\mathrm{Si}_{3} \mathrm{~N}_{4}$ insulation and standard processing techniques.

The gain measurements were carried out using a variant of a multisection cavity technique successfully applied on GaAs-based QC lasers ${ }^{20}$. Samples were processed in four-section ridges: an absorbing section, a light-bulb section (A), an insulating (or middle) section (M) and an amplifier section (B). To decrease the electrical cross-talk between sections, the heavily doped cladding region was removed over a width of about $20 \mu \mathrm{m}$ by a dry etching process. During the whole measurement, the absorbing section was grounded to damp optical feedback and prevent the device from oscillating. Section A, used as a light bulb, was driven above the onset of negative differential resistance. In this manner, the injection process is non-resonant and does not only populate the upper state but also the quasi-continuum of states lying above, providing a relatively broad emission spectrum. The middle section is biased to an intermediate bias where no significant current is injected, but also where the ground state of the active region, lifted above the Fermi energy of the injector, is essentially empty, preventing reabsorption. Electroluminescence measurements were carried out in step-scan mode using a Nicolet 860 Fourier-transform infrared spectrometer. A selected sequence of pulses, combined with a phase-sensitive detection, enables the simultaneous measurement of the electroluminescence spectrum of section A amplified by section B, minus the direct electroluminescence of section $\mathrm{B}$, and the emission of section $\mathrm{A}$ alone. The net modal gain of the amplifier section can then be directly extracted from this measurement as shown in the Supplementary Information.

The gain computations were carried out, in a first step, by computing the band-structure self-consistently assuming thermal populations. In a second step, the electron population was computed for each level through rate equations, assuming for both electron injection and extraction a resonant tunnelling formalism based on density matrices ${ }^{10,21}$. In this computation, the population lifetimes were computed assuming bulk optical phonon emission. In-plane scattering times were taken from the measured width of the 
electroluminescence: $0.13 \mathrm{ps}(10 \mathrm{meV})$ for sample $\mathrm{N} 258$ and $0.16 \mathrm{ps}(8 \mathrm{meV})$ in sample N123. The influence of the populations on the upper and lower laser states on the band alignment was neglected, that is, only the thermal populations were taken into account. Once the populations were obtained, the second-order gain was computed including the non-parabolicity effects. The second-order gain at frequency $\omega$ can be written as ${ }^{7}$ :

$$
g(\omega)=\frac{e^{2}\left|z_{21}\right|^{2} \epsilon^{2}}{\varepsilon_{0} n_{r} c \hbar^{2} \omega} \sum_{k} \frac{\gamma_{k}^{1}\left(f_{k}^{22}-f_{k_{+}}^{11}\right)+\gamma_{k}^{2}\left(f_{k_{-}}^{22}-f_{k}^{11}\right)}{(\epsilon-\hbar \omega)^{2}+\left(\gamma_{k}^{1}+\gamma_{k}^{2}\right)^{2}},
$$

where $z_{21}$ is the dipole matrix element, $\epsilon$ is the intersubband transition energy, $f_{k}^{i i}$ is the distribution function of state $i$ at wavevector $k$ and $\gamma_{k}^{i}$ is the homogeneous broadening of state $i$ at wavevector $k ; e$ is the electron charge, $\varepsilon_{0}$ is the vacuum permittivity, $n_{r}$ is the refractive index, $c$ is the speed of light and $\hbar$ is Planck's constant divided by $2 \pi$. The gain expression consists of two terms: an emission term $f_{k}^{22}-f_{k_{+}}^{11}$ and an absorption term $f_{k_{-}}^{22}-f_{k}^{11}$, where the scattering-assisted transitions appear through the special wavevectors $k_{ \pm}$ shown in Fig. 1a,b. To include the non-parabolicity, all energies, including the wavevectors $k_{+}$and $k_{-}$at which the distribution functions are evaluated, are now computed assuming an energy-dependent effective mass with the form $m^{*}(E)=m^{*}(0)\left(1+E / E_{\mathrm{G}}\right)$, where $E_{\mathrm{G}}=0.8 \mathrm{eV}$ is the energy gap.

Received 26 September 2006; accepted 24 January 2007; published 1 April 2007.

References

1. Esaki, L. \& Tsu, R. Superlattice and negative differential conductivity in semiconductors. IBM J. Res. Develop. 14, 61-65 (1970)

2. Bloch, F. Uber die quantenmechhanik der elektronen in kristallgittern. Z. Phys. 52, 555-600 (1928).

3. Zener, C. A theory of the electrical breakdown of solid dielectrics. Proc. R. Soc. A 145, 523-529 (1934).

4. Ktitorov, S. A., Simin, G. S. \& Sindalovskii, V. Y. Bragg reflections and the high-frequency conductivity of an electronic solid-state plasma. Fiz. tverd. Tela. 13, 2230-2233 (1971).

5. Ignatov, A. A. \& Romanov, Y. A. Nonlinear electromagnetic properties of semiconductors with a superlattice. Phys. Status Solidi B 73, 327-333 (1976).

6. Choi, K. K., Levine, B. F., Malik, R. J., Walker, J. \& Bethea, C. G. Periodic negative conductance by sequential resonant tunneling through an expanding high-field superlattice domain. Phys. Rev. B 35, $4172-4175(1987)$

7. Willenberg, H., Döhler, G. H. \& Faist, J. Intersubband gain in a Bloch oscillator and quantum cascade laser. Phys. Rev. B 67, 085315 (2003)
8. Wacker, A. Gain in quantum cascade lasers and superlattices: A quantum transport theory. Phys. Rev. B 66, 085326 (2002).

9. Faist, J. et al. Quantum cascade laser. Science 264, 553-556 (1994)

10. Sirtori, C. et al. Resonant tunneling in quantum cascade lasers. IEEE J. Quantum Electron. 34, 1722-1729 (1998).

11. Feldmann, J. et al. Optical investigation of Bloch oscillations in a semiconductor superlattice. Phys. Rev. B 46, 7252-7255 (1992).

12. Waschke, C. et al. Coherent submillimeter-wave emission from Bloch oscillations in a semiconductor superlattice. Phys. Rev. Lett. 70, 3319-3322 (1993).

13. Sekine, N. \& Hirakawa, K. Dispersive terahertz gain of a nonclassical oscillator: Bloch oscillation in semiconductor superlattices. Phys. Rev. Lett. 94, 057408-057412 (2005).

14. Unterrainer, K. et al. Inverse Bloch oscillator: Strong terahertz-photocurrent resonances at the Bloch frequency. Phys. Rev. Lett. 76, 2973-2976 (1996).

15. Savvidis, P., Kolasa, B., Lee, G. \& Allen, S. Resonant crossover of terahertz loss to the gain of a Bloch oscillating inas/alsb superlattice. Phys. Rev. Lett. 92, 196802 (2004).

16. Imamoglu, A. \& Ram, R. J. Semiconductor lasers without population inversion. Opt. Lett. 19 , 1744-1746 (1994)

17. Faist, J. et al. Quantum cascade lasers without intersubband population inversion. Phys. Rev. Lett. 76, 411-414 (1996).

18. Gorfinkel, V., Luryi, S. \& Gelmont, B. Theory of gain spectra for quantum cascade lasers and temperature dependence of their characteristics at low and moderate carrier concentrations. IEEE J. Quantum Electron. 32, 1995-2003 (1996).

19. Sirtori, C. et al. Mid-infrared $(8.5 \mu \mathrm{m})$ semiconductor lasers operating at room temperature. IEEE Photon. Technol. Lett. 9, 294-296 (1997).

20. Barbieri, S. et al. Gain measurements on gaas-based quantum cascade lasers using a two-section cavity technique. IEEE J. Quantum Electron. 36, 736-741 (2000).

21. Kazarinov, R. F. \& Suris, R. A. Electric and electromagnetic properties of semiconductors with a superlattice. Sov. Phys. Semicond. 6, 120-131 (1972).

\section{Acknowledgements}

This work was supported by the Swiss National Science Foundation and the National Center of Competence in Research, Quantum Photonics.

Correspondence and requests for materials should be addressed to R.T

Supplementary Information accompanies this paper on www.nature.com/naturephysics.

\section{Author contributions}

The structures were designed by J.F. and grown by M.G. and N.H. using molecular beam epitaxy. T.G. fabricated the samples, worked on the measurement technique and measured the samples together with N.S. The theoretical work and the gain calculations were done by R.T. who also wrote the manuscript together with J.F., the head of the group in which the work was carried out.

\section{Competing financial interests}

The authors declare no competing financial interests.

Reprints and permission information is available online at http://npg.nature.com/reprintsandpermissions/ 